\title{
SOLVABILITY IN THE SENSE OF SEQUENCES FOR SOME NON-FREDHOLM ELLIPTIC PROBLEMS
}

\author{
Vitaly Volpert ${ }^{1}$, Vitali Vougalter ${ }^{2}$ \\ ${ }^{1}$ Institute Camille Jordan, UMR 5208 CNRS, University Lyon 1 \\ Villeurbanne, 69622, France \\ e-mail: volpert@math.univ-lyon1.fr \\ ${ }^{2}$ Department of Mathematics and Applied Mathematics, University of Cape Town \\ Private Bag, Rondebosch 7701, South Africa \\ e-mail: Vitali.Vougalter@uct.ac.za
}

\begin{abstract}
We establish solvability in the sense of sequences in the appropriate $H^{2}$ spaces for certain linear nonhomogeneous elliptic problems involving Schrödinger type operators without Fredholm property using the technique developed in our preceding work [22]. We show the existence of bounded solutions for certain nonlinear Lippmann-Schwinger equations.
\end{abstract}

AMS Subject Classification: 35J10, 35P10, 35P25

Key words: solvability conditions, non Fredholm operators, elliptic problems.

\section{Introduction}

Solvability conditions for linear and nonlinear elliptic problems with non Fredholm operators were studied extensively in recent years. Most of the articles on the subject concern a single equation of the second order (see [12], [13], [14], [17], [18], [20]). One of the few exceptions was the linearized Cahn-Hilliard problem studied in [16]. This equation of the fourth order can be trivially related to the system of two nonhomogeneous equations of second order. The first equation in it would be the standard Poisson equation having an explicit solution decaying fast enough at infinity under the appropriate assumptions on its right side. The second equation in the system would be the nonhomogeneous Schrödinger equation. In [19] we exploit the similar ideas to obtain the solvability conditions for the linearized Cahn-Hilliard equation of the sixth order involving the bi-Laplacian operator. Work [21] deals with establishing the existence of stationary solutions for certain systems of nonlinear integro-differential equations involving operators without Fredholm property. In [23] we seek the standing wave solutions of certain 
systems of nonlinear, nonlocal equations using the fixed point technique for the map involving non Fredholm operators.

One of the important questions about equations with non-Fredholm operators concerns their solvability. We will study it in the following setting. Let $f_{n}$ be a sequence of functions in the image of the operator $A$, such that $f_{n} \rightarrow f$ in $L^{2}\left(\mathbb{R}^{d}\right)$ as $n \rightarrow \infty$. Denote by $u_{n}$ a sequence of functions from $H^{2}\left(\mathbb{R}^{d}\right)$ such that

$$
A u_{n}=f_{n}, n \in \mathbb{N} .
$$

Since the operator $A$ does not satisfy the Fredholm property, then the sequence $u_{n}$ may not be convergent. We will call a sequence $u_{n}$ such that $A u_{n} \rightarrow f$ a solution in the sense of sequences of equation $A u=f$ (see [10]). If this sequence converges to a function $u_{0}$ in the norm of the space, then $u_{0}$ is a solution of this equation. Solution in the sense of sequences is equivalent in this sense to the usual solution. However, in the case of non-Fredholm operators this convergence may not hold or it can occur in some weaker sense. In this case, solution in the sense of sequences may not imply the existence of the usual solution. In this work we will find sufficient conditions of equivalence of solutions in the sense of sequences and the usual solutions. In the other words, the conditions on sequences $f_{n}$ under which the corresponding sequences $u_{n}$ are strongly convergent.

In the first part of the present article we consider the system of two Schrödinger type equations, solvability conditions for which were obtained in [15], namely

$$
\left\{\begin{array}{l}
\Delta u+a(x) u+\alpha u+b(x) v=f(x), \\
\Delta v+c(x) v+\beta v=g(x)
\end{array}\right.
$$

where $\alpha, \beta \geq 0$ are constants, $x \in \mathbb{R}^{3}$, the potential functions $a(x)$ and $c(x)$ are considered to be shallow and short-range and satisfy the conditions analogous to those used in works [12], [13], [14].

Assumption 1. The potential function $a(x): \mathbb{R}^{3} \rightarrow \mathbb{R}$ satisfies the estimate

$$
|a(x)| \leq \frac{C}{1+|x|^{3.5+\varepsilon}}
$$

with some $\varepsilon>0$ and $x=\left(x_{1}, x_{2}, x_{3}\right) \in \mathbb{R}^{3}$ a.e. such that

$$
4^{\frac{1}{9}} \frac{9}{8}(4 \pi)^{-\frac{2}{3}}\|a\|_{L^{\infty}\left(\mathbb{R}^{3}\right)}^{\frac{1}{9}}\|a\|_{L^{\frac{4}{3}\left(\mathbb{R}^{3}\right)}}^{\frac{8}{9}}<1
$$

and

$$
\sqrt{c_{H L S}}\|a\|_{L^{\frac{3}{2}\left(\mathbb{R}^{3}\right)}}<4 \pi
$$

and the requirements for $c(x)$ here are exactly the same as for $a(x)$. 
Here $C$ stands for a finite positive constant and $c_{H L S}$ is given on p.98 of [6] is the constant in the Hardy-Littlewood-Sobolev inequality

$$
\left|\int_{\mathbb{R}^{3}} \int_{\mathbb{R}^{3}} \frac{f_{1}(x) f_{1}(y)}{|x-y|^{2}} d x d y\right| \leq c_{H L S}\left\|f_{1}\right\|_{L^{\frac{3}{2}\left(\mathbb{R}^{3}\right)}}^{2}, \quad f_{1} \in L^{\frac{3}{2}}\left(\mathbb{R}^{3}\right) .
$$

Here and further down the norm of a function $f_{1} \in L^{p}\left(\mathbb{R}^{3}\right), 1 \leq p \leq \infty$ is designated as $\left\|f_{1}\right\|_{L^{p}\left(\mathbb{R}^{3}\right)}$. We will be using

$$
\left(f_{1}(x), f_{2}(x)\right)_{L^{2}\left(\mathbb{R}^{3}\right)}:=\int_{\mathbb{R}^{3}} f_{1}(x) \bar{f}_{2}(x) d x
$$

with a slight abuse of notations when the functions involved in this inner product are not necessarily square integrable. For instance, if $f_{1}(x) \in L^{1}\left(\mathbb{R}^{3}\right)$ and $f_{2}(x)$ is bounded, like the functions involved in the orthogonality conditions of Theorems 4 and 8 , then the integral in the right side of (1.3) still makes sense. The sphere of radius $r$ in the space of three dimensions centered at the origin will be denoted by $S_{r}^{3}$. For system (1.1) we write down the corresponding sequence of systems with $n \in \mathbb{N}$ :

$$
\left\{\begin{array}{l}
\Delta u+a(x) u+\alpha u+b_{n}(x) v=f_{n}(x), \\
\Delta v+c(x) v+\beta v=g_{n}(x)
\end{array}\right.
$$

Let us make the appropriate assumptions on the right sides and the coefficients of (1.4).

Assumption 2. Let $n \in \mathbb{N}, f_{n}(x): \mathbb{R}^{3} \rightarrow \mathbb{R}, f_{n}(x) \in L^{2}\left(\mathbb{R}^{3}\right)$ and $|x| f_{n}(x) \in$ $L^{1}\left(\mathbb{R}^{3}\right)$, such that $f_{n}(x) \rightarrow f(x)$ in $L^{2}\left(\mathbb{R}^{3}\right)$ and $|x| f_{n}(x) \rightarrow|x| f(x)$ in $L^{1}\left(\mathbb{R}^{3}\right)$ as $n \rightarrow \infty$. The requirements for $g_{n}(x)$ and $g(x)$ here are exactly the same ones as for $f_{n}(x)$ and $f(x)$.

Assumption 3. Let $n \in \mathbb{N}, b_{n}(x): \mathbb{R}^{3} \rightarrow \mathbb{R}, b_{n}(x) \in L^{\infty}\left(\mathbb{R}^{3}\right)$ and $|x| b_{n}(x) \in$ $L^{2}\left(\mathbb{R}^{3}\right)$, such that $b_{n}(x) \rightarrow b(x)$ in $L^{\infty}\left(\mathbb{R}^{3}\right)$ and $|x| b_{n}(x) \rightarrow|x| b(x)$ in $L^{2}\left(\mathbb{R}^{3}\right)$ as $n \rightarrow \infty$.

By means of the decay of the potential functions at infinity stated explicitly in Assumption 1, for the Schrödinger operators involved in the left sides of equations of systems (1.1) and (1.4)

$$
h_{\alpha}:=-\Delta-a(x)-\alpha \quad \text { and } \quad l_{\beta}:=-\Delta-c(x)-\beta, \quad \alpha, \beta \geq 0
$$

on $L^{2}\left(\mathbb{R}^{3}\right)$, their essential spectra concide with the semi-axes $[-\alpha, \infty)$ and $[-\beta, \infty)$ respectively (see e.g. [4]), such that $h_{\alpha}$ and $l_{\beta}$ do not possess finite dimensional isolated kernels. Therefore, the Fredholm alternative theorem fails to work for equations of systems (1.1) and (1.4). The operators $h_{0}$ and $l_{0}$ correspond to the case when the constants $\alpha$ and $\beta$ vanish. 
Due to Assumption 1, the Schrödinger operators $h_{\alpha}$ and $l_{\beta}$ defined in (1.5) are self-adjoint and unitarily equivalent to $-\Delta-\alpha$ and $-\Delta-\beta$ on $L^{2}\left(\mathbb{R}^{3}\right)$ respectively via the wave operators (see [1], [5], [8], Lemma 2.3 of [12]). Their functions of the continuous spectra satisfy

$$
l_{0} \varphi_{k}(x)=k^{2} \varphi_{k}(x), k \in \mathbb{R}^{3}, \quad h_{0} \eta_{q}(x)=q^{2} \eta_{q}(x), q \in \mathbb{R}^{3},
$$

the Lippmann-Schwinger equations for the perturbed plane waves (see e.g. [7] p.98)

$$
\begin{aligned}
& \varphi_{k}(x)=\frac{e^{i k x}}{(2 \pi)^{\frac{3}{2}}}+\frac{1}{4 \pi} \int_{\mathbb{R}^{3}} \frac{e^{i|k||x-y|}}{|x-y|}\left(c \varphi_{k}\right)(y) d y, \\
& \eta_{q}(x)=\frac{e^{i q x}}{(2 \pi)^{\frac{3}{2}}}+\frac{1}{4 \pi} \int_{\mathbb{R}^{3}} \frac{e^{i|q||x-y|}}{|x-y|}\left(a \eta_{q}\right)(y) d y,
\end{aligned}
$$

the orthogonality relations with $k, q \in \mathbb{R}^{3}$ :

$$
\left(\varphi_{k}(x), \varphi_{q}(x)\right)_{L^{2}\left(\mathbb{R}^{3}\right)}=\delta(k-q), \quad\left(\eta_{k}(x), \eta_{q}(x)\right)_{L^{2}\left(\mathbb{R}^{3}\right)}=\delta(k-q)
$$

and form the complete systems in $L^{2}\left(\mathbb{R}^{3}\right)$. The functions $\varphi_{0}(x)$ and $\eta_{0}(x)$ correspond to the case when the wave vectors are equal to zero. We will be using the appropriate Sobolev space for vector functions equipped with the norm

$$
\left\|\left(u_{1}, u_{2}\right)^{T}\right\|_{H^{2}\left(\mathbb{R}^{3}, \mathbb{R}^{2}\right)}^{2}:=\sum_{k=1}^{2}\left\|u_{k}\right\|_{H^{2}\left(\mathbb{R}^{3}\right)}^{2}=\sum_{k=1}^{2}\left\{\left\|u_{k}\right\|_{L^{2}\left(\mathbb{R}^{3}\right)}^{2}+\left\|\Delta u_{k}\right\|_{L^{2}\left(\mathbb{R}^{3}\right)}^{2}\right\} .
$$

Our first main result is as follows.

Theorem 4. Let Assumptions 1, 2 and 3 hold. If $\beta>0$, let

$$
\left(g_{n}(x), \varphi_{k}(x)\right)_{L^{2}\left(\mathbb{R}^{3}\right)}=0, \quad k \in S_{\sqrt{\beta}}^{3} \quad \text { a.e. }
$$

hold for $n \in \mathbb{N}$. If $\beta=0$, let

$$
\left(g_{n}(x), \varphi_{0}(x)\right)_{L^{2}\left(\mathbb{R}^{3}\right)}=0, \quad n \in \mathbb{N} .
$$

Then the second equation in (1.4) admits a unique solution $v_{0, n}(x) \in H^{2}\left(\mathbb{R}^{3}\right)$. Furthermore, if $\alpha>0$, let

$$
\left(f_{n}(x)-b_{n}(x) v_{0, n}(x), \eta_{q}(x)\right)_{L^{2}\left(\mathbb{R}^{3}\right)}=0, \quad q \in S_{\sqrt{\alpha}}^{3} \quad \text { a.e. }
$$

hold for $n \in \mathbb{N}$. If $\alpha=0$, let

$$
\left(f_{n}(x)-b_{n}(x) v_{0, n}(x), \eta_{0}(x)\right)_{L^{2}\left(\mathbb{R}^{3}\right)}=0, \quad n \in \mathbb{N} .
$$

Then systems (1.4) and (1.1) possess unique solutions $\left(u_{0, n}, v_{0, n}\right)^{T} \in H^{2}\left(\mathbb{R}^{3}, \mathbb{R}^{2}\right)$ and $\left(u_{0}, v_{0}\right)^{T} \in H^{2}\left(\mathbb{R}^{3}, \mathbb{R}^{2}\right)$ respectively, such that $\left(u_{0, n}, v_{0, n}\right)^{T} \rightarrow\left(u_{0}, v_{0}\right)^{T}$ in $H^{2}\left(\mathbb{R}^{3}, \mathbb{R}^{2}\right)$ as $n \rightarrow \infty$. 
In the second part of the article we consider

$$
\xi_{k}(x)=\frac{e^{i k x}}{(2 \pi)^{\frac{3}{2}}}-\frac{1}{4 \pi} \int_{\mathbb{R}^{3}} \frac{e^{i|k||x-y|}}{|x-y|} F\left(\xi_{k}(y), y\right) d y, \quad k \in \mathbb{R}^{3},
$$

which is the generalized Lippmann-Schwinger equation. Its solutions will satisfy

$$
-\Delta \xi_{k}+F\left(\xi_{k}(x), x\right)=k^{2} \xi_{k}(x), \quad k \in \mathbb{R}^{3} .
$$

Understanding the properties of solutions of equations of (1.13) type plays a crucial role when studying problems of nonlinear optics (see e.g. [9]). Let us make the following assumption on the term involved in the integral part of (1.13), which in principal can be nonlinear.

Assumption 5. Function $F(u, x): \mathbb{C} \times \mathbb{R}^{3} \rightarrow \mathbb{C}$ is such that

$$
\left|F\left(u_{1}, x\right)-F\left(u_{2}, x\right)\right| \leq \mu(x)\left|u_{1}-u_{2}\right| \quad \text { for any } u_{1,2} \in \mathbb{C}, x \in \mathbb{R}^{3}
$$

with the scalar function $\mu(x): \mathbb{R}^{3} \rightarrow \mathbb{R}^{+}$satisfying inequality (1.2) and $F(0, x)=$ 0 for any $x \in \mathbb{R}^{3}$.

The following functional space appeared actively in the studies of the spectral properties of non Fredholm operators (see e.g. [18])

$$
\tilde{W}^{2, \infty}\left(\mathbb{R}^{3}\right):=\left\{w(x): \mathbb{R}^{3} \rightarrow \mathbb{C} \mid w, \nabla w, \Delta w \in L^{\infty}\left(\mathbb{R}^{3}\right)\right\} .
$$

We will be using (1.15) in the present work as well. Note that (1.15) differs from the standard $W^{2, \infty}\left(\mathbb{R}^{3}\right)$ space since here we do not require all the partial derivatives up to the second order of a function to be bounded. Our second main result is as follows.

Theorem 6. Let Assumption 5 hold. Then for each $k \in \mathbb{R}^{3}$ equation (1.13) admits a unique nontrivial solution $\xi_{k}(x) \in \tilde{W}^{2, \infty}\left(\mathbb{R}^{3}\right)$.

The final part of the article is devoted to the studies of the diffusion equation with convection, solvability conditions for which were established in [18]. Let us consider

$$
\Delta u+v \cdot \nabla u+c(x) u=f(x), \quad x \in \mathbb{R}^{3},
$$

where $p(x)$ is the liquid pressure and the velocity field $v(x)=-\nabla p(x)$.

Assumption 7. The liquid pressure is a function $p(x): \mathbb{R}^{3} \rightarrow \mathbb{R}$, such that $p(x) \in W^{2, \infty}\left(\mathbb{R}^{3}\right)$.

Similarly to the first part of the article, we write down the sequence of equations corresponding to (1.16) as

$$
\Delta u+v \cdot \nabla u+c(x) u=f_{n}(x), \quad x \in \mathbb{R}^{3}, n \in \mathbb{N} .
$$


The homogeneous equation formally adjoint for both (1.16) and (1.17) will be

$$
\Delta w-\operatorname{div}(v w)+c(x) w=0 .
$$

Analogously to [18], let us introduce the change of variables for both equations (1.16) and (1.17) as

$$
u(x):=z(x) e^{\frac{p(x)}{2}},
$$

which will relate these equations to

$$
H_{a} z=G \quad \text { and } \quad H_{a} z=G_{n}
$$

respectively. Here the right sides

$$
G(x):=-f(x) e^{-\frac{p(x)}{2}} \quad \text { and } \quad G_{n}(x):=-f_{n}(x) e^{-\frac{p(x)}{2}} .
$$

According to [18], the Schrödinger operator involved in the left sides of equations (1.20) is given by $H_{a}:=-\Delta+W_{0}(x)-a$ with a shallow and short-range potential $W_{0}(x):=\frac{(\nabla p)^{2}}{4}-\frac{\Delta p}{2}-c(x)+a$ and a constant $a \geq 0$. Similarly to the first part of the article, due to the assumptions on the scalar potential, the functions of the continuous spectrum satisfy

$$
\left(-\Delta+W_{0}(x)\right) \gamma_{k}(x)=k^{2} \gamma_{k}(x), \quad k \in \mathbb{R}^{3},
$$

the Lippmann-Schwinger integral equation

$$
\gamma_{k}(x)=\frac{e^{i k x}}{(2 \pi)^{\frac{3}{2}}}-\frac{1}{4 \pi} \int_{\mathbb{R}^{3}} \frac{e^{i|k||x-y|}}{|x-y|}\left(W_{0} \gamma_{k}\right)(y) d y, \quad k \in \mathbb{R}^{3},
$$

the orthogonality relations analogous to (1.7) and form the complete system in $L^{2}\left(\mathbb{R}^{3}\right)$. Our final main result is as follows.

Theorem 8. Let Assumption 7 hold, $n \in \mathbb{N}$, functions $f_{n}(x)$ satisfy Assumption 2, the potential $W_{0}(x)$ satisfies Assumption 1 and for an arbitrary solution of problem (1.18) $w(x) \in \tilde{W}^{2, \infty}\left(\mathbb{R}^{3}\right)$ the orthogonality conditions

$$
\left(f_{n}(x), w(x)\right)_{L^{2}\left(\mathbb{R}^{3}\right)}=0
$$

hold. Then equations (1.17) and (1.16) admit unique solutions $u_{n}(x) \in H^{2}\left(\mathbb{R}^{3}\right)$ and $u(x) \in H^{2}\left(\mathbb{R}^{3}\right)$ respectively, such that $u_{n}(x) \rightarrow u(x)$ in $H^{2}\left(\mathbb{R}^{3}\right)$ as $n \rightarrow \infty$.

Understanding the spectral properties of non Fredholm operators is very important, for instance when establishing the existence in certain functional spaces of stationary and travelling wave solutions of reaction-diffusion equations (see e.g. [2], [3], [11], [18]). 


\section{Solvability in the sense of sequences for a system of equations}

Proof of Theorem 4. By means of Theorem 4 of [22] via orthogonality relations (1.9) and (1.10) the second equations in systems (1.4) and (1.1) admit unique solutions $v_{0, n}(x) \in H^{2}\left(\mathbb{R}^{3}\right), n \in \mathbb{N}$ and $v_{0}(x) \in H^{2}\left(\mathbb{R}^{3}\right)$ respectively, such that $v_{0, n}(x) \rightarrow v_{0}(x)$ in $H^{2}\left(\mathbb{R}^{3}\right)$ as $n \rightarrow \infty$. Hence we can rewrite the first equation in system (1.4) as

$$
\Delta u+a(x) u+\alpha u=f_{n}(x)-b_{n}(x) v_{0, n}(x), \quad n \in \mathbb{N}
$$

and the first equation in system (1.1) as

$$
\Delta u+a(x) u+\alpha u=f(x)-b(x) v_{0}(x) .
$$

Note that the right side of (2.22) belongs to $L^{2}\left(\mathbb{R}^{3}\right)$ due to Assumptions 2 and 3 and the fact that $v_{0, n}(x) \in L^{2}\left(\mathbb{R}^{3}\right)$. Let us estimate the norm from above as

$$
\left\|b_{n} v_{0, n}-b v_{0}\right\|_{L^{2}\left(\mathbb{R}^{3}\right)} \leq\left\|\left(b_{n}-b\right) v_{0, n}\right\|_{L^{2}\left(\mathbb{R}^{3}\right)}+\left\|b\left(v_{0, n}-v_{0}\right)\right\|_{L^{2}\left(\mathbb{R}^{3}\right)} .
$$

Clearly, for the first term in the right side of (2.24) we have the upper bound

$$
\left\|\left(b_{n}-b\right) v_{0, n}\right\|_{L^{2}\left(\mathbb{R}^{3}\right)} \leq\left\|b_{n}-b\right\|_{L^{\infty}\left(\mathbb{R}^{3}\right)}\left\|v_{0, n}\right\|_{L^{2}\left(\mathbb{R}^{3}\right)} \rightarrow 0, \quad n \rightarrow \infty
$$

due to Assumption 3 and the fact that $v_{0, n}(x) \rightarrow v_{0}(x)$ in $L^{2}\left(\mathbb{R}^{3}\right)$. For the second term in the right side of (2.24) we have the trivial inequality

$$
\left\|b\left(v_{0, n}-v_{0}\right)\right\|_{L^{2}\left(\mathbb{R}^{3}\right)} \leq\|b\|_{L^{\infty}\left(\mathbb{R}^{3}\right)}\left\|v_{0, n}-v_{0}\right\|_{L^{2}\left(\mathbb{R}^{3}\right)} \rightarrow 0, \quad n \rightarrow \infty
$$

using Assumption 3 as well. Therefore, for the norm of the difference of the right sides of equations (2.22) and (2.23) we have the bound

$$
\left\|f_{n}-b_{n} v_{0, n}-\left[f-b v_{0}\right]\right\|_{L^{2}\left(\mathbb{R}^{3}\right)} \leq\left\|f_{n}-f\right\|_{L^{2}\left(\mathbb{R}^{3}\right)}+\left\|b v_{0}-b_{n} v_{0, n}\right\|_{L^{2}\left(\mathbb{R}^{3}\right)} \rightarrow 0
$$

as $n \rightarrow \infty$ via Assumption 2 and the norm estimates obtained above. Hence

$$
f_{n}(x)-b_{n}(x) v_{0, n}(x) \rightarrow f(x)-b(x) v_{0}(x)
$$

in $L^{2}\left(\mathbb{R}^{3}\right)$ as $n \rightarrow \infty$. By means of the Schwarz inequality along with Assumptions 2 and 3 for $v_{0, n}(x) \in L^{2}\left(\mathbb{R}^{3}\right)$ we arrive at

$$
\left\||x|\left(f_{n}-b_{n} v_{0, n}\right)\right\|_{L^{1}\left(\mathbb{R}^{3}\right)} \leq\left\||x| f_{n}\right\|_{L^{1}\left(\mathbb{R}^{3}\right)}+\left\||x| b_{n}\right\|_{L^{2}\left(\mathbb{R}^{3}\right)}\left\|v_{0, n}\right\|_{L^{2}\left(\mathbb{R}^{3}\right)}<\infty,
$$

such that $|x|\left(f_{n}(x)-b_{n}(x) v_{0, n}(x)\right) \in L^{1}\left(\mathbb{R}^{3}\right)$. We estimate the norm of the difference as

$$
\left\||x| b_{n} v_{0, n}-|x| b v_{0}\right\|_{L^{1}\left(\mathbb{R}^{3}\right)} \leq\left\||x|\left(b_{n}-b\right) v_{0, n}\right\|_{L^{1}\left(\mathbb{R}^{3}\right)}+\left\||x| b\left(v_{0, n}-v_{0}\right)\right\|_{L^{1}\left(\mathbb{R}^{3}\right)} .
$$


Via the Schwarz inequality along with Assumption 3 for $v_{0, n}(x) \in L^{2}\left(\mathbb{R}^{3}\right)$ and $v_{0, n}(x) \rightarrow v_{0}(x)$ in $L^{2}\left(\mathbb{R}^{3}\right)$ as $n \rightarrow \infty$ we obtain

$$
\begin{gathered}
\left\||x|\left(b_{n}-b\right) v_{0, n}\right\|_{L^{1}\left(\mathbb{R}^{3}\right)} \leq\left\||x| b_{n}-|x| b\right\|_{L^{2}\left(\mathbb{R}^{3}\right)}\left\|v_{0, n}\right\|_{L^{2}\left(\mathbb{R}^{3}\right)} \rightarrow 0, \quad n \rightarrow \infty \\
\|\| x\left|b\left(v_{0, n}-v_{0}\right)\left\|_{L^{1}\left(\mathbb{R}^{3}\right)} \leq\right\|\right| x \mid b\left\|_{L^{2}\left(\mathbb{R}^{3}\right)}\right\| v_{0, n}-v_{0} \|_{L^{2}\left(\mathbb{R}^{3}\right)} \rightarrow 0, \quad n \rightarrow \infty
\end{gathered}
$$

such that $|x| b_{n}(x) v_{0, n}(x) \rightarrow|x| b(x) v_{0}(x)$ in $L^{1}\left(\mathbb{R}^{3}\right)$ as $n \rightarrow \infty$. Evidently,

$$
\begin{gathered}
\left\||x|\left(f_{n}-b_{n} v_{0, n}\right)-|x|\left(f-b v_{0}\right)\right\|_{L^{1}\left(\mathbb{R}^{3}\right)} \leq\left\||x| f_{n}-|x| f\right\|_{L^{1}\left(\mathbb{R}^{3}\right)}+ \\
+\left\||x| b v_{0}-|x| b_{n} v_{0, n}\right\|_{L^{1}\left(\mathbb{R}^{3}\right)} \rightarrow 0
\end{gathered}
$$

as $n \rightarrow \infty$ due to Assumption 2 and the estimate above. Thus,

$$
|x|\left(f_{n}(x)-b_{n}(x) v_{0, n}(x)\right) \rightarrow|x|\left(f(x)-b(x) v_{0}(x)\right)
$$

in $L^{1}\left(\mathbb{R}^{3}\right)$ as $n \rightarrow \infty$ and orthogonality relations (1.11) and (1.12) hold according to our assumption. Theorem 4 of [22] implies that equations (2.22) and (2.23) admit unique solutions $u_{0, n}(x) \in H^{2}\left(\mathbb{R}^{3}\right), n \in \mathbb{N}$ and $u_{0}(x) \in H^{2}\left(\mathbb{R}^{3}\right)$ respectively, such that $u_{0, n}(x) \rightarrow u_{0}(x)$ in $H^{2}\left(\mathbb{R}^{3}\right)$ as $n \rightarrow \infty$. Norm definition (1.8) implies that $\left(u_{0, n}, v_{0, n}\right)^{T} \rightarrow\left(u_{0}, v_{0}\right)^{T}$ in $H^{2}\left(\mathbb{R}^{3}, \mathbb{R}^{2}\right)$ as $n \rightarrow \infty$.

\section{The existence of solutions for the generalized Lippmann- Schwinger equation}

Proof of Theorem 6. Obviously, by means of Assumption 5 we have

$$
|F(u, x)| \leq \mu(x)|u|, \quad \text { for any } u \in \mathbb{C}, x \in \mathbb{R}^{3} .
$$

In order to study the existence of solutions of (1.13), we introduce the auxiliary problem

$$
u(x)=\frac{e^{i k x}}{(2 \pi)^{\frac{3}{2}}}-\frac{1}{4 \pi} \int_{\mathbb{R}^{3}} \frac{e^{i|k||x-y|}}{|x-y|} F(v(y), y) d y, \quad k \in \mathbb{R}^{3} .
$$

Let us choose an arbitrary $v(x) \in L^{\infty}\left(\mathbb{R}^{3}\right)$. Then via (3.25) for the right side of (3.26) we have the estimate from above in the absolute value as

$$
\frac{1}{(2 \pi)^{\frac{3}{2}}}+\frac{1}{4 \pi}\|v\|_{L^{\infty}\left(\mathbb{R}^{3}\right)} \sup _{x \in \mathbb{R}^{3}} \int_{\mathbb{R}^{3}} \frac{\mu(y)}{|x-y|} d y<\infty,
$$

which is guaranteed by Lemma 2.1 of [12] for $\mu(x)$ satisfying inequality (1.2) due to Assumption 5. Therefore, the left side of equation (3.26) is determined uniquely and $u(x) \in L^{\infty}\left(\mathbb{R}^{3}\right)$. Hence, the auxiliary problem (3.26) defines the map $T: L^{\infty}\left(\mathbb{R}^{3}\right) \rightarrow L^{\infty}\left(\mathbb{R}^{3}\right)$ with $u(x)=T v(x)$. 
Let us choose arbitrarily $v_{1,2}(x) \in L^{\infty}\left(\mathbb{R}^{3}\right)$. Then $u_{1,2}(x):=T v_{1,2}(x) \in$ $L^{\infty}\left(\mathbb{R}^{3}\right)$, such that

$$
u_{1}(x)-u_{2}(x)=-\frac{1}{4 \pi} \int_{\mathbb{R}^{3}} \frac{e^{i|k||x-y|}}{|x-y|}\left\{F\left(v_{1}(y), y\right)-F\left(v_{2}(y), y\right)\right\} d y .
$$

Assumption 5 yields

$$
\left|u_{1}(x)-u_{2}(x)\right| \leq \frac{1}{4 \pi} \int_{\mathbb{R}^{3}} \frac{\mu(y)}{|x-y|}\left|v_{1}(y)-v_{2}(y)\right| d y .
$$

Therefore,

$$
\left\|T v_{1}-T v_{2}\right\|_{L^{\infty}\left(\mathbb{R}^{3}\right)} \leq \frac{1}{4 \pi} \sup _{x \in \mathbb{R}^{3}} \int_{\mathbb{R}^{3}} \frac{\mu(y)}{|x-y|} d y\left\|v_{1}-v_{2}\right\|_{L^{\infty}\left(\mathbb{R}^{3}\right)},
$$

where $\mu(x)$ satisfies inequality (1.2) via Assumption 5. Lemma 2.1 of [12] gives us that the constant involved in the right side of estimate (3.27)

$$
\frac{1}{4 \pi} \sup _{x \in \mathbb{R}^{3}} \int_{\mathbb{R}^{3}} \frac{\mu(y)}{|x-y|} d y<1
$$

such that the map $T: L^{\infty}\left(\mathbb{R}^{3}\right) \rightarrow L^{\infty}\left(\mathbb{R}^{3}\right)$ is a strict contraction and therefore, admits a unique fixed point $\xi_{k}(x) \in L^{\infty}\left(\mathbb{R}^{3}\right)$, which is the unique solution in $L^{\infty}\left(\mathbb{R}^{3}\right)$ of problem (1.13) for a given $k \in \mathbb{R}^{3}$. Note that this solution is nontrivial since via Assumption 5 for any $x \in \mathbb{R}^{3}$ we have $F(0, x)=0$. Inequality (3.25) yields

$$
\left|F\left(\xi_{k}(x), x\right)\right| \leq \mu(x)\left|\xi_{k}(x)\right|
$$

where $\mu(x) \in L^{\infty}\left(\mathbb{R}^{3}\right)$ since it has to satisfy inequality (1.2) according to our assumption. Thus, $F\left(\xi_{k}(x), x\right) \in L^{\infty}\left(\mathbb{R}^{3}\right)$. From equation (1.14) we easily deduce that $\Delta \xi_{k}(x) \in L^{\infty}\left(\mathbb{R}^{3}\right)$ as well. A trivial calculation using (1.13) yields that $\nabla \xi_{k}(x)$ equals to

$$
\frac{e^{i k x}}{(2 \pi)^{\frac{3}{2}}} i k-\frac{1}{4 \pi} \int_{\mathbb{R}^{3}}\left[e^{i|k||x-y|} \frac{x-y}{|x-y|^{2}} i|k|-e^{i|k||x-y|} \frac{x-y}{|x-y|^{3}}\right] F\left(\xi_{k}(y), y\right) d y .
$$

By means of (3.29) along with (3.28) we have

$$
\int_{\mathbb{R}^{3}} \frac{\left|F\left(\xi_{k}(y), y\right)\right|}{|x-y|} d y \leq\left\|\xi_{k}\right\|_{L^{\infty}\left(\mathbb{R}^{3}\right)} \sup _{x \in \mathbb{R}^{3}} \int_{\mathbb{R}^{3}} \frac{\mu(y)}{|x-y|} d y<\infty .
$$

Evidently,

$$
\sup _{x \in \mathbb{R}^{3}} \int_{\mathbb{R}^{3}} \frac{\mu(y)}{|x-y|^{2}} d y<\infty
$$


which is guaranteed by Lemma A3 of [18] with $\mu(x) \in L^{\infty}\left(\mathbb{R}^{3}\right) \cap L^{\frac{4}{3}}\left(\mathbb{R}^{3}\right)$ due to Assumption 5. Then (3.29) yields

$$
\int_{\mathbb{R}^{3}} \frac{\left|F\left(\xi_{k}(y), y\right)\right|}{|x-y|^{2}} d y \leq\left\|\xi_{k}\right\|_{L^{\infty}\left(\mathbb{R}^{3}\right)} \sup _{x \in \mathbb{R}^{3}} \int_{\mathbb{R}^{3}} \frac{\mu(y)}{|x-y|^{2}} d y<\infty
$$

and therefore, $\nabla \xi_{k}(x) \in L^{\infty}\left(\mathbb{R}^{3}\right)$. Thus, $\xi_{k}(x) \in \tilde{W}^{2, \infty}\left(\mathbb{R}^{3}\right)$, which completes the proof of the theorem.

\section{Solvability in the sense of sequences for the diffusion equation with convection}

Proof of Theorem 8. Under the assumptions of Theorem 8 by means of Theorem 3 of [18] equation (1.17) admits a unique solution $u_{n}(x) \in H^{2}\left(\mathbb{R}^{3}\right), n \in \mathbb{N}$. Assumption 2 along with part a) of Lemma 7 of [22] under orthogonality conditions (1.21) imply

$$
(f(x), w(x))_{L^{2}\left(\mathbb{R}^{3}\right)}=0 .
$$

Via Theorem 3 of [18], equation (1.16) has a unique solution $u(x) \in H^{2}\left(\mathbb{R}^{3}\right)$. Clearly, $G_{n}(x) \in L^{2}\left(\mathbb{R}^{3}\right), n \in \mathbb{N}$ as a product of two functions one of which is square integrable and another is bounded by Assumption 7. For the norm of the difference of the right sides of equations (1.20) we have the trivial equality

$$
\left\|G_{n}-G\right\|_{L^{2}\left(\mathbb{R}^{3}\right)}=\sqrt{\int_{\mathbb{R}^{3}}\left|f_{n}(x)-f(x)\right|^{2} e^{-p(x)} d x} .
$$

Its right side can be bounded above due to Assumption 7 by

$$
C\left\|f_{n}(x)-f(x)\right\|_{L^{2}\left(\mathbb{R}^{3}\right)} \rightarrow 0
$$

as $n \rightarrow \infty$ by Assumption 2. Here and further down $C$ will stand for a finite positive constant. Hence $G_{n}(x) \rightarrow G(x)$ in $L^{2}\left(\mathbb{R}^{3}\right)$ as $n \rightarrow \infty$. By applying Assumptions 2 and 7 we easily derive $|x| G_{n}(x) \in L^{1}\left(\mathbb{R}^{3}\right), n \in \mathbb{N}$ and

$$
\begin{gathered}
\left\||x| G_{n}(x)-|x| G(x)\right\|_{L^{1}\left(\mathbb{R}^{3}\right)}=\int_{\mathbb{R}^{3}}|x|\left|f_{n}(x)-f(x)\right| e^{-\frac{p(x)}{2}} d x \leq \\
\leq C\left\||x| f_{n}(x)-|x| f(x)\right\|_{L^{1}\left(\mathbb{R}^{3}\right)} \rightarrow 0
\end{gathered}
$$

as $n \rightarrow \infty$, such that $|x| G_{n}(x) \rightarrow|x| G(x)$ in $L^{1}\left(\mathbb{R}^{3}\right)$ as $n \rightarrow \infty$. Let us define

$$
w_{k}(x):=e^{-\frac{p(x)}{2}} \gamma_{k}(x)
$$

with $k \in S_{\sqrt{a}}^{3}$ a.e. if the constant $a>0$ and $k=0$ if $a=0$. As discussed in [18], functions $w_{k}(x) \in \tilde{W}^{2, \infty}\left(\mathbb{R}^{3}\right)$ and are solutions of problem (1.18). Therefore, by means of (1.21)

$$
\left(f_{n}(x), w_{k}(x)\right)_{L^{2}\left(\mathbb{R}^{3}\right)}=0, \quad n \in \mathbb{N},
$$


which implies

$$
\left(G_{n}(x), \gamma_{k}(x)\right)_{L^{2}\left(\mathbb{R}^{3}\right)}=0, \quad k \in S_{\sqrt{a}}^{3} \text { a.e., } n \in \mathbb{N}
$$

when $a>0$ and

$$
\left(G_{n}(x), \gamma_{0}(x)\right)_{L^{2}\left(\mathbb{R}^{3}\right)}=0, \quad n \in \mathbb{N}
$$

when $a=0$. Then via Theorem 4 of [22], the first and the second equations in (1.20) admit unique solutions $z(x) \in H^{2}\left(\mathbb{R}^{3}\right)$ and $z_{n}(x) \in H^{2}\left(\mathbb{R}^{3}\right), n \in \mathbb{N}$ respectively and $z_{n}(x) \rightarrow z(x)$ in $H^{2}\left(\mathbb{R}^{3}\right)$ as $n \rightarrow \infty$. Let us use (1.19) along with Assumption 7 to estimate

$$
\left\|u_{n}-u\right\|_{L^{2}\left(\mathbb{R}^{3}\right)}=\sqrt{\int_{\mathbb{R}^{3}}\left|z_{n}(x)-z(x)\right|^{2} e^{p(x)} d x} \leq C\left\|z_{n}-z\right\|_{L^{2}\left(\mathbb{R}^{3}\right)} \rightarrow 0
$$

as $n \rightarrow \infty$. From (1.19) we easily deduce

$$
\begin{gathered}
\Delta u_{n}=e^{\frac{p}{2}} \Delta z_{n}+e^{\frac{p}{2}} \nabla z_{n} . \nabla p+e^{\frac{p}{2}} \frac{z_{n}}{2} \Delta p+e^{\frac{p}{2}} \frac{z_{n}}{4}(\nabla p)^{2}, \\
\Delta u=e^{\frac{p}{2}} \Delta z+e^{\frac{p}{2}} \nabla z \cdot \nabla p+e^{\frac{p}{2}} \frac{z}{2} \Delta p+e^{\frac{p}{2}} \frac{z}{4}(\nabla p)^{2} .
\end{gathered}
$$

The estimates below will be based on Assumption 7 .

$$
\begin{gathered}
\left\|e^{\frac{p}{2}} \Delta z_{n}-e^{\frac{p}{2}} \Delta z\right\|_{L^{2}\left(\mathbb{R}^{3}\right)}=\sqrt{\int_{\mathbb{R}^{3}}\left|\Delta z_{n}(x)-\Delta z(x)\right|^{2} e^{p(x)} d x} \leq \\
\leq C\left\|\Delta z_{n}-\Delta z\right\|_{L^{2}\left(\mathbb{R}^{3}\right)} \rightarrow 0
\end{gathered}
$$

as $n \rightarrow \infty$. Since

$$
\left|e^{\frac{p}{2}} \nabla z_{n} . \nabla p-e^{\frac{p}{2}} \nabla z \cdot \nabla p\right| \leq C\left|\nabla z_{n}-\nabla z\right|,
$$

using the Schwarz inequality we obtain

$$
\begin{gathered}
\left\|e^{\frac{p}{2}} \nabla z_{n} \cdot \nabla p-e^{\frac{p}{2}} \nabla z \cdot \nabla p\right\|_{L^{2}\left(\mathbb{R}^{3}\right)} \leq C\left\|\nabla z_{n}-\nabla z\right\|_{L^{2}\left(\mathbb{R}^{3}\right)} \leq \\
\leq C \sqrt{\left\|\Delta z_{n}-\Delta z\right\|_{L^{2}\left(\mathbb{R}^{3}\right)}\left\|z_{n}-z\right\|_{L^{2}\left(\mathbb{R}^{3}\right)}} \rightarrow 0
\end{gathered}
$$

as $n \rightarrow \infty$. Obviously,

$$
\left|e^{\frac{p}{2}} \frac{z_{n}}{2} \Delta p-e^{\frac{p}{2}} \frac{z}{2} \Delta p\right| \leq C\left|z_{n}-z\right|,
$$

such that

$$
\left\|e^{\frac{p}{2}} \frac{z_{n}}{2} \Delta p-e^{\frac{p}{2}} \frac{z}{2} \Delta p\right\|_{L^{2}\left(\mathbb{R}^{3}\right)} \leq C\left\|z_{n}-z\right\|_{L^{2}\left(\mathbb{R}^{3}\right)} \rightarrow 0
$$


as $n \rightarrow \infty$. Finally,

$$
\left|e^{\frac{p}{2}} \frac{z_{n}}{4}(\nabla p)^{2}-e^{\frac{p}{2}} \frac{z}{4}(\nabla p)^{2}\right| \leq C\left|z_{n}-z\right|
$$

which yields

$$
\left\|e^{\frac{p}{2}} \frac{z_{n}}{4}(\nabla p)^{2}-e^{\frac{p}{2}} \frac{z}{4}(\nabla p)^{2}\right\|_{L^{2}\left(\mathbb{R}^{3}\right)} \leq C\left\|z_{n}-z\right\|_{L^{2}\left(\mathbb{R}^{3}\right)} \rightarrow 0
$$

as $n \rightarrow \infty$. Identities (2.30) and (2.31) along with the estimates obtained above give us $\Delta u_{n} \rightarrow \Delta u$ in $L^{2}\left(\mathbb{R}^{3}\right)$ as $n \rightarrow \infty$. Thus, we arrive at $u_{n}(x) \rightarrow u(x)$ in $H^{2}\left(\mathbb{R}^{3}\right)$ as $n \rightarrow \infty$.

\section{References}

[1] H.L. Cycon, R.G. Froese, W. Kirsch, B. Simon, Schrödinger Operators with Application to Quantum Mechanics and Global Geometry, Springer-Verlag, Berlin (1987).

[2] A. Ducrot, M. Marion, V. Volpert, Systemes de réaction-diffusion sans propriété de Fredholm, CRAS, 340 (2005), 659-664.

[3] A. Ducrot, M. Marion, V. Volpert, Reaction-diffusion problems with non Fredholm operators, Advances Diff. Equations, 13, No. 11-12 (2008), 11511192.

[4] B.L.G. Jonsson, M. Merkli, I.M. Sigal, F. Ting, Applied Analysis, In preparation.

[5] T. Kato, Wave operators and similarity for some non-selfadjoint operators, Math. Ann., 162 (1965/1966), 258-279.

[6] E. Lieb, M. Loss, Analysis. Graduate Studies in Mathematics, 14, American Mathematical Society, Providence (1997).

[7] M. Reed, B. Simon, Methods of Modern Mathematical Physics, III: Scattering Theory, Academic Press (1979).

[8] I. Rodnianski, W. Schlag, Time decay for solutions of Schrödinger equations with rough and time-dependent potentials, Invent. Math., 155, No. 3 (2004), 451-513.

[9] V. Serov, Inverse Born approximation for the nonlinear two-dimensional Schrödinger operator, Inverse Problems, 23, No. 3 (2007), 1259-1270. 
[10] V. Volpert. Elliptic partial differential equations. Volume 1. Fredholm theory of elliptic problems in unbounded domains. Birkhäuser, 2011.

[11] V. Volpert, B. Kazmierczak, M. Massot, Z.Peradzynski, Solvability conditions for elliptic problems with non-Fredholm operators, Appl.Math., 29, No. 2 (2002), 219-238.

[12] V. Vougalter, V. Volpert, Solvability conditions for some non Fredholm operators, Proc. Edinb. Math. Soc. (2), 54, No. 1 (2011), 249-271.

[13] V. Vougalter, V. Volpert. On the solvability conditions for some non Fredholm operators, Int. J. Pure Appl. Math., 60, No. 2 (2010), 169-191.

[14] V. Vougalter, V. Volpert. Solvability relations for some non Fredholm operators, Int. Electron. J. Pure Appl. Math., 2, No. 1 (2010), 75-83.

[15] V. Vougalter, V. Volpert. Solvability conditions for some systems with non Fredholm operators, Int. Electron. J. Pure Appl. Math., 2, No. 3 (2010), 183187.

[16] V. Volpert, V. Vougalter. On the solvability conditions for a linearized CahnHilliard equation, Rend. Istit. Mat. Univ. Trieste, 43, (2011), 1-9.

[17] V. Vougalter, V. Volpert. On the existence of stationary solutions for some nonFredholm integro-differential equations, Doc. Math., 16, (2011), 561-580.

[18] V. Vougalter, V. Volpert. On the solvability conditions for the diffusion equation with convection terms, Commun. Pure Appl. Anal., 11, No. 1 (2012), 365-373.

[19] V. Vougalter, V. Volpert. Solvability conditions for a linearized Cahn-Hilliard equation of sixth order, Math. Model. Nat. Phenom., 7, No. 2 (2012), 146154.

[20] V. Vougalter, V. Volpert. Solvability conditions for some linear and nonlinear non-Fredholm elliptic problems, Anal. Math. Phys., 2, No.4 (2012), 473-496.

[21] V. Volpert, V. Vougalter. On the existence of stationary solutions for some systems of non-Fredholm integro-differential equations, Disc., Nonlin. and Complex., 1, No.2 (2012), 85-98.

[22] V. Volpert, V. Vougalter. Solvability in the sense of sequences for some non Fredholm operators, Preprint (2013).

[23] V. Vougalter. Solvability conditions for some systems of nonlinear nonFredholm elliptic equations, Disc., Nonlin. and Complex., 2, No.2 (2013), $1-6$. 